
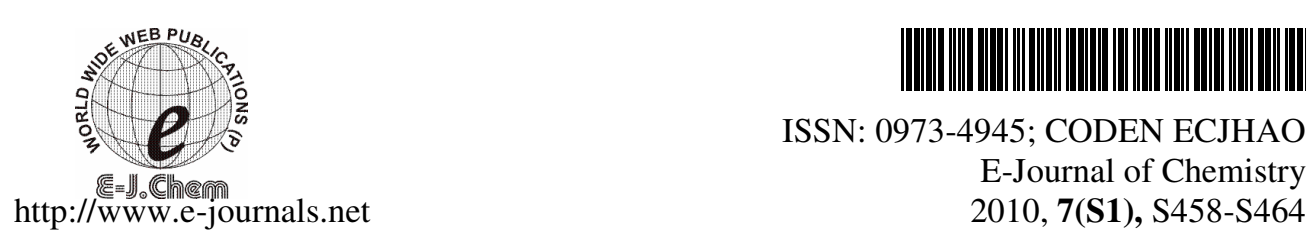

ISSN: 0973-4945; CODEN ECJHAO

E-Journal of Chemistry

2010, 7(S1), S458-S464

\title{
Synthesis and Antimicrobial Activity of Some Novel Schiff Bases Containing 1,2,4-Triazole-3-thione
}

\author{
M. M. ÇALIŞIR, B. KOÇYİĞİT-KAYMAKÇIOGLU*, B. ÖZBEK ${ }^{\S}$ and G. ÖTÜK \\ Marmara University, Faculty of Pharmacy \\ Department of Pharmaceutical Chemistry, İstanbul- Turkey \\ ${ }^{\S}$ İstanbul University, Faculty of Pharmacy \\ Department of Pharmaceutical Microbiology, İstanbul- Turkey \\ bkaymakcioglu@marmara.edu.tr
}

Received 25 December 2009; Accepted 24 February 2010

\begin{abstract}
A series of Schiff bases (4a-j) were prepared by reacting 4-amino-5(1-phenylethyl)-2,4-dihydro-3H-1,2,4-triazole-3-thione and substituted aromatic aldehydes. The chemical structures were confirmed by means of UV, ${ }^{1} \mathrm{H}$ NMR, IR, MS(API-ES) spectral data and elemental analysis. All the synthesized compounds were screened for their antimicrobial activity. Among the tested compounds, Schiff bases with 4-OH and 4- $\mathrm{NO}_{2}$ substituent exhibited remarkable antimicrobial activity against all the tested microorganism.
\end{abstract}

Keywords: 1,2,4-Triazole-3-thione, Schiff bases, Antimicrobial activity.

\section{Introduction}

The compounds carrying azomethine functional group $-\mathrm{C}=\mathrm{N}$ - which are known as Schiff bases gain importance in medicinal and pharmaceutical field due to the most versatile organic synthetic intermediates and also showing a broad range of biological activities such as antituberculosis ${ }^{1,2}$, anticancer ${ }^{3,4}$, analgesic and antiinflamatory $y^{5,6}$, anticonvulsant ${ }^{7-9}$, antibacterial and antifungal ${ }^{10,11}$ activities.

1,2,4-Triazoles have also been found to possess a wide spectrum of pharmacological activities $^{12-18}$. The 1,2,4-triazole nucleus has been incorporated in to a wide variety of therapeutically interesting molecules to transform them in to better drugs. The drugs such as fluconazole and itraconazole are the best examples for potent antifungal molecules possessing triazole nucleus ${ }^{19}$.

The development of antimicrobial resistance has increased in this century and there is a need of synthesis of new antimicrobial agents which will be more selective, potent and less toxic compared to the used drugs in clinical treatment. These observations prompted us to 
synthesize a series of Schiff bases containing 1,2,4-triazole ring system on the same molecule due to the importance of triazole and Schiff bases in biological systems by means of antimicrobial activity.

\section{Experimental}

All the chemicals and solvents were purchased from Merck, Aldrich, and Fluka. Melting points were determined with Kleinfeld SMP-II and were uncorrected. UV spectra were recorded on a Schimadzu UV 2100S spectrophotometer. The IR spectra were recorded on a Schimadzu FTIR $8400 \mathrm{~S}$ spectrometry. ${ }^{1} \mathrm{H}$ NMR spectra were recorded in DMSO on a Bruker Avance-DPX-400 spectrometer in DMSO- $d_{6}$ and chemical shifts were given in $\delta$ ppm with tetramethylsilane (TMS). The splitting patterns of ${ }^{1} \mathrm{H}$ NMR were designed as follows: $\mathrm{s}$ : singlet, $\mathrm{d}$ : doublet, $\mathrm{t}$ : triplet, q: quarlet, m. multiplet. Elemental analysis was performed on Leco CHNS-932 analyzer.

Preparation of 4-amino-5-(1-phenylethyl)-2,4-dihydro-3H-1,2,4-triazole-3-thione (3)

Equimolar mixture of 2-phenylpropanoic acide (1) and thiocarbohydrazide (2) $(0.1 \mathrm{~mol})$ was heated in an oil bath at $130{ }^{\circ} \mathrm{C}$ for $2 \mathrm{~h}$. The fused mass obtained was dispersed with hot water to obtain the 4 -aminotriazole (3). The product was recrystallized from methanol.

UV: $\lambda_{\text {maks }}(\mathrm{nm})(\log \varepsilon): 254,(4.54) ; 200(4.98)$. IR $v_{\text {maks }}\left(\mathrm{cm}^{-1}\right): 3321,3184(\mathrm{~N}-\mathrm{H}), 1138$ $(\mathrm{C}=\mathrm{S}) .{ }^{1} \mathrm{H}$ NMR (DMSO-d 6 , $\left.400 \mathrm{MHz}\right) \delta(\mathrm{ppm}): 1.54\left(\mathrm{~d}, 3 \mathrm{H}, \mathrm{CH}_{3}\right) 4.36(\mathrm{~m}, 1 \mathrm{H}, \mathrm{CH}), 5.42$ (s, 2H, $\mathrm{NH}_{2}$ ), 7.43-7.50 ( m, 5H, Ar), 13.60 (s, 1H, NH). Anal. Calcd. For $\mathrm{C}_{10} \mathrm{H}_{12} \mathrm{~N}_{4} \mathrm{~S}: \mathrm{C}$, 54.52; H, 5.49; N, 25.43; S, 14.56. Found: C, 54.41; H, 5.30; N, 24.88; S, 14.20.

Preparation of 4-[[(4-aryl)methylidene]amino]-5-(1-phenylethyl)-2,4-dihydro-3H1,2,4-triazole-3-thione $(\mathbf{4 a - j})$

A solution of 0.005 mol of substituted aldehydes in ethanol was added a solution of 0.005 mol of 4-aminotriazole in $50 \mathrm{~mL}$ ethanol. The mixture was refluxed on a water bath for 2-2.5 h. After cooling the mixture, the precipitate was filtred, dried and recrystallized from ethanol.

\section{4-\{[4-(Dimethylamino)phenyl]methylidene\}amino)-5-(1-phenylethyl)-2,4-dihydro-} 3H-1,2,4-triazole-3-thione (4a)

UV: $\lambda_{\text {maks }}(\mathrm{nm})(\log \varepsilon): 361(4.66) ; 244,(4.43)$. IR $v_{\text {maks }}\left(\mathrm{cm}^{-1}\right): 3381(\mathrm{~N}-\mathrm{H}), 1182(\mathrm{C}=\mathrm{S})$. ${ }^{1} \mathrm{H}$ NMR (DMSO-d $\left.d_{6}, 400 \mathrm{MHz}\right) \delta(\mathrm{ppm}): 1.57\left(\mathrm{~d}, 3 \mathrm{H}, \mathrm{CH}_{3}\right), 3.02\left(\mathrm{~s}, 6 \mathrm{H}, \mathrm{N}\left(\mathrm{CH}_{3}\right)_{2}\right), 4.34$ (q, 1H, CH ), 6.77 ( d, J: $8.92 \mathrm{~Hz}, 2 \mathrm{H}, \mathrm{Ar}), 7.19-7.25$ ( m, 5H, Ar ), 7.58 ( d, J: $8.90 \mathrm{~Hz}, 2 \mathrm{H}$, Ar) $9.22(\mathrm{~s}, 1 \mathrm{H}, \mathrm{CH}), 13.78(\mathrm{~s}, 1 \mathrm{H}, \mathrm{NH})$, Anal. Calcd. For $\mathrm{C}_{19} \mathrm{H}_{21} \mathrm{~N}_{5} \mathrm{~S}: \mathrm{C}, 64.93 ; \mathrm{H}, 6.02$; N, 19.93; S, 9.12. Found: C, 63.98; H, 5.98; N, 19.90; S, 9.10

4-\{[(3,4-Dimethoxyphenyl)methylidene]amino\}-5-(1-phenylethyl)-2,4-dihydro-3H1,2,4-triazole-3-thione (4b)

UV: $\lambda_{\text {maks }}(\mathrm{nm}),(\log \varepsilon): 323$ (4.16) ; $285(4.01)$. IR $v_{\text {maks }}\left(\mathrm{cm}^{-1}\right): 3294(\mathrm{~N}-\mathrm{H}), 1180(\mathrm{C}=\mathrm{S})$. ${ }^{1} \mathrm{H}$ NMR (DMSO- $\left.d_{6}, 400 \mathrm{MHz}\right) \delta(\mathrm{ppm}): 1.60\left(\mathrm{~d}, 3 \mathrm{H}, \mathrm{CH}_{3}\right), 3.60\left(\mathrm{~s}, 6 \mathrm{H}, \mathrm{OCH}_{3}\right) 4.40(\mathrm{q}$, $1 \mathrm{H}, \mathrm{CH}), 7.18-7.47(\mathrm{~m}, 8 \mathrm{H}, \mathrm{Ar}), 9.74(\mathrm{~s}, 1 \mathrm{H}, \mathrm{CH}), 13.78(\mathrm{~s}, 1 \mathrm{H}, \mathrm{NH})$. Anal. Calcd. for $\mathrm{C}_{19} \mathrm{H}_{20} \mathrm{~N}_{4} \mathrm{O}_{2} \mathrm{~S}$ : C, $61.94 ; \mathrm{H}, 5.47 ; \mathrm{N}, 15.21 ; \mathrm{S}, 8.70$. Found: C, $61.90 ; \mathrm{H}, 5.32 ; \mathrm{N}, 15.10$; S, 8.35. MS(API-ES), $m / z: 369\left(\mathrm{M}^{+}+1\right) ; 368\left(\mathrm{M}^{+}\right) ; 367\left(\mathrm{M}^{+}-1\right), 204$.

4-\{[(Pyridin-4-yl)methylidene]amino $\}-5-(1-$ phenylethyl)-2,4-dihydro-3H-1,2,4triazole-3-thione $(\mathbf{4 c})$

UV: $\lambda_{\text {maks }}(\mathrm{nm}),(\log \varepsilon): 357$ (4.34) ; 285 (4.01). IR $v_{\text {maks }}\left(\mathrm{cm}^{-1}\right) 3095(\mathrm{~N}-\mathrm{H}), 1190(\mathrm{C}=\mathrm{S})$. ${ }^{1} \mathrm{H}$ NMR (DMSO-d 6 , $\left.400 \mathrm{MHz}\right) \delta(\mathrm{ppm}): 1.60\left(\mathrm{~d}, 3 \mathrm{H}, \mathrm{CH}_{3}\right), 4.49(\mathrm{q}, 1 \mathrm{H}, \mathrm{CH}), 7.18-8.00$ (m, 9H, Ar), 10.27 ( s, 1H, CH ), 14.04 ( s, 1H, NH ). Anal. Calcd. for $\mathrm{C}_{16} \mathrm{H}_{15} \mathrm{~N}_{5} \mathrm{~S}: \mathrm{C}, 62.11$; H, 4.89; N, 22.64; S, 10.36. Found: C, 62.00; H, 4.10; N, 22.60; S, 10.09. 
4-\{[(4-Hydroxyphenyl)methylidene ]amino\}-5-(1-phenylethyl)-2,4-dihydro-3H-1,2,4 -triazole-3-thione $(\mathbf{4 d})$

UV: $\lambda_{\text {maks }}(\mathrm{nm}),(\log \varepsilon): 323$ (4.16) ; 269 (3.78). IR $v_{\text {maks }}\left(\mathrm{cm}^{-1}\right) 3051(\mathrm{OH}$ and N-H), 1194 $(\mathrm{C}=\mathrm{S}) .{ }^{1} \mathrm{H}$ NMR $\left(\mathrm{DMSO}-d_{6}, 400 \mathrm{MHz}\right) \delta(\mathrm{ppm}): 1.56\left(\mathrm{~d}, 3 \mathrm{H}, \mathrm{CH}_{3}\right), 4.37(\mathrm{q}, 1 \mathrm{H}, \mathrm{CH}), 6.88$ (d, J: $7.79 \mathrm{~Hz}, 2 \mathrm{H}, \mathrm{Ar}), 7.19-7.28$ (m, 5H, Ar), $7.63(\mathrm{~d}, J: 7.80 \mathrm{~Hz}, 2 \mathrm{H}, \mathrm{Ar}), 9.43(\mathrm{~s}, 1 \mathrm{H}$, $\mathrm{OH}), 10.35(\mathrm{~s}, 1 \mathrm{H}, \mathrm{CH}), 13.85(\mathrm{~s}, 1 \mathrm{H}, \mathrm{NH})$. Anal. Calcd. For $\mathrm{C}_{17} \mathrm{H}_{16} \mathrm{~N}_{4} \mathrm{OS}: \mathrm{C}, 62.94 ; \mathrm{H}$, 4.97; N, 17.27; S, 9.88. Found: C, 62.00; H, 4.40; N, 17.60; S, 10.09.

4-\{[(4-Nitrophenyl)methylidene]amino\}-5-(1-phenylethyl)-2,4-dihydro-3H-1,2,4triazole-3-thione $(\mathbf{4 e})$

UV: $\lambda_{\text {maks }}(\mathrm{nm}),(\log \varepsilon): 359$ (3.58) ; 283 (4.17). IR $v_{\text {maks }}\left(\mathrm{cm}^{-1}\right) 3080(\mathrm{~N}-\mathrm{H}), 1168(\mathrm{C}=\mathrm{S}) .{ }^{1} \mathrm{H}-$ NMR (DMSO- $\left.d_{6}, 400 \mathrm{MHz}\right) \delta(\mathrm{ppm}): 1.60\left(\mathrm{~d}, 3 \mathrm{H}, \mathrm{CH}_{3}\right), 4.59(\mathrm{q}, 1 \mathrm{H}, \mathrm{CH}), 7.20-7.30(\mathrm{~m}, 5 \mathrm{H}$, Ar ), 8.10 (d, J: $8.87 \mathrm{~Hz}, 2 \mathrm{H}, \mathrm{Ar}), 8.30$ (d, J: $8.83 \mathrm{~Hz}, 2 \mathrm{H}, \mathrm{Ar}), 10.27$ ( s, 1H, CH ), 14.00 ( s, 1H, $\mathrm{NH}$ ). Anal. Calcd. for $\mathrm{C}_{17} \mathrm{H}_{15} \mathrm{~N}_{5} \mathrm{O}_{2} \mathrm{~S}: \mathrm{C}, 57.78 ; \mathrm{H}, 4.28 ; \mathrm{N}, 19.82 ; \mathrm{S}, 9.07$. Found: C, 58.01; H, 4.25; N, 19.80; S, 8.90. MS (API-ES), m/z: $354\left(\mathrm{M}^{+}+1\right) ; 353\left(\mathrm{M}^{+}\right) ; 352\left(\mathrm{M}^{+}-1\right), 204$.

4-\{[(4-Chlorophenyl)methylidene]amino\}-5-(1-phenylethyl)-2,4-dihydro-3H-1,2,4triazole-3-thione $(\mathbf{4 f})$

UV: $\lambda_{\text {maks }}(\mathrm{nm}),(\log \varepsilon): 328(3.29) ; 262$ (4.12). IR $v_{\text {maks }}\left(\mathrm{cm}^{-1}\right) 3100(\mathrm{~N}-\mathrm{H}), 1166(\mathrm{C}=\mathrm{S})$. ${ }^{1} \mathrm{H}$ NMR (DMSO- $\left.d_{6}, 400 \mathrm{MHz}\right) \delta(\mathrm{ppm}): 1.59$ ( d, $\left.3 \mathrm{H}, \mathrm{CH}_{3}\right), 4.44(\mathrm{q}, 1 \mathrm{H}, \mathrm{CH}), 7.18-7.30$ (m, 5H, Ar), 7.60 ( d, J: $8.51 \mathrm{~Hz}, 2 \mathrm{H}, \mathrm{Ar}), 7.81$ ( d, J: $8.55 \mathrm{~Hz}, 2 \mathrm{H}, \mathrm{Ar}), 9.84$ (s, 1H, CH), 13.74 (s, $1 \mathrm{H}, \mathrm{NH})$. Anal. Anal. Calcd. For $\mathrm{C}_{17} \mathrm{H}_{15} \mathrm{ClN}_{4} \mathrm{~S}$ : C, 59.56; H, 4.41; N,16.34; S, 9.35. Found: C, 59.41; H, 4.30; N, 16.88; S, 9.20.

4-\{[(3-Ethoxy-4-hydroxyphenyl)methylidene]amino\}-5-(1-phenylethyl)-2,4-dihidro3H-1,2,4-triazol-3-thione (4g)

UV: $\lambda_{\text {maks }}(\mathrm{nm}),(\log \varepsilon): 328(3,89) ; 252(3.98)$. IR $v_{\text {maks }}\left(\mathrm{cm}^{-1}\right) 3095(\mathrm{OH}$ and N-H), 1178 $(\mathrm{C}=\mathrm{S}) .{ }^{1} \mathrm{H}$ NMR (DMSO- $\left.d_{6}, 400 \mathrm{MHz}\right) \delta(\mathrm{ppm}): 1.06-1.58\left(\mathrm{~m}, 6 \mathrm{H}, \mathrm{CH}_{3}\right), 4.05-4.10(3 \mathrm{H}, \mathrm{q}$, $\mathrm{CH}$ and $\left.\mathrm{CH}_{2}\right), 6.87-7.43(\mathrm{~m}, 8 \mathrm{H}, \mathrm{Ar}), 8.55(\mathrm{~s}, 1 \mathrm{H}, \mathrm{OH}), 9.62(\mathrm{~s}, 1 \mathrm{H}, \mathrm{CH}), 13.90(\mathrm{~s}, 1 \mathrm{H}, \mathrm{NH})$. Anal. Calcd. for $\mathrm{C}_{19} \mathrm{H}_{20} \mathrm{~N}_{4} \mathrm{O}_{2} \mathrm{~S}: \mathrm{C}, 61.94 ; \mathrm{H}, 5.47 ; \mathrm{N}, 15.21 ; \mathrm{S}, 8.70$. Found: C, 61.05; H, $5.10 ; \mathrm{N}, 15.20 ; \mathrm{S}, 8.45$.

4-\{[(2-Hydroxyphenyl)methylidene]amino\}-5-(1-phenylethyl)-2,4-dihydro-3H-1,2,4 -triazole-3-thione $(\mathbf{4 h})$

UV: $\lambda_{\text {maks }}(\mathrm{nm}),(\log \varepsilon): 357(4,34) ; 294(4,37)$. IR $v_{\text {maks }}\left(\mathrm{cm}^{-1}\right) 3095(\mathrm{OH}$ and N-H), 1178 $(\mathrm{C}=\mathrm{S}) .{ }^{1} \mathrm{H}$ NMR (DMSO- $\left.d_{6}, 400 \mathrm{MHz}\right) \delta(\mathrm{ppm}): 1.58\left(\mathrm{~d}, 3 \mathrm{H}, \mathrm{CH}_{3}\right.$ ), 4.42 (q, 1H, CH ), 6.90$7.74(\mathrm{~m}, 9 \mathrm{H}, \mathrm{Ar}), 9.99(\mathrm{~s}, 1 \mathrm{H}, \mathrm{OH}), 10.28(\mathrm{~s}, 1 \mathrm{H}, \mathrm{CH}), 13.90(\mathrm{~s}, 1 \mathrm{H}, \mathrm{NH})$. Anal. Calcd. for $\mathrm{C}_{17} \mathrm{H}_{16} \mathrm{~N}_{4} \mathrm{OS}$ : C, 62.94, H, 4.97; N, 17.27; S, 9.88. Found: C, 62.05; H, 4.40; N, 17.64; S, 10.08 .

4-\{[(4-Bromophenyl)methylidene]amino\}-5-(1-phenylethyl)-2,4-dihydro-3H-1,2,4triazole-3-thione $(\mathbf{4 i})$

UV: $\lambda_{\text {maks }}(\mathrm{nm}),(\log \varepsilon): 359(3,58) ; 283(4,17)$. IR $v_{\text {maks }}\left(\mathrm{cm}^{-1}\right) 3095(\mathrm{~N}-\mathrm{H}), 1172(\mathrm{C}=\mathrm{S})$. ${ }^{1} \mathrm{H}$ NMR (DMSO- $\left.d_{6}, 400 \mathrm{MHz}\right) \delta(\mathrm{ppm}): 1.58\left(\mathrm{~d}, 3 \mathrm{H}, \mathrm{CH}_{3}\right), 4.44(\mathrm{q}, 1 \mathrm{H}, \mathrm{CH}), 7.13-7.31(\mathrm{~m}$, $5 \mathrm{H}, \mathrm{Ar}), 7.58(\mathrm{~d}, \mathrm{~J}: 8.51 \mathrm{~Hz}, 2 \mathrm{H}, \mathrm{Ar}), 7.80$ (d, J: $8.55 \mathrm{~Hz}, 2 \mathrm{H}, \mathrm{Ar}), 9.82(\mathrm{~s}, 1 \mathrm{H}, \mathrm{CH}), 14.00$ (s, 1H, triazol NH). Anal. Calcd. For $\mathrm{C}_{17} \mathrm{H}_{15} \mathrm{BrN}_{4} \mathrm{~S}$ : C, 52.72; H, 3.90; N, 14.47; S, 8.28. Found: C, 53.00; H, 3.98; N, $14.90 ; \mathrm{S}, 8.10$. 
4-\{[(4-Benzyloxyphenyl)methylidene]amino\}-5-(1-phenylethyl)-2,4-dihydro-3H-1,2, 4-triazole-3-thione (4j)

UV: $\lambda_{\text {maks }}(\mathrm{nm}),(\log \varepsilon): 203(4,45) ; 327(4,48)$. IR $v_{\text {maks }}\left(\mathrm{cm}^{-1}\right) 3200(\mathrm{~N}-\mathrm{H}), 1166(\mathrm{C}=\mathrm{S}) .{ }^{1} \mathrm{H}-$ NMR (DMSO- $\left.d_{6}, 400 \mathrm{MHz}\right) \delta(\mathrm{ppm}): 2.00\left(\mathrm{~d}, 3 \mathrm{H}, \mathrm{CH}_{3}\right), 3.35$ (s, $\left.2 \mathrm{H}, \mathrm{CH}_{2}\right), 4.60(\mathrm{q}, 1 \mathrm{H}, \mathrm{CH})$, 7.50-8.10 (m, 14H, Ar), $10.20(\mathrm{~s}, 1 \mathrm{H}, \mathrm{CH}), 13.60(\mathrm{~s}, 1 \mathrm{H}, \mathrm{NH})$. Anal. Calcd. For $\mathrm{C}_{24} \mathrm{H}_{22} \mathrm{~N}_{4} \mathrm{OS}: \mathrm{C}$, 69.54; H, 5.35; N, 13.52; S, 7.74. Found: C, 68.25; H, 4.98; N, 13.20; S, 7.10.

\section{Antimicrobial activity}

Antimicrobial activity against Staphylococcus aureus ATCC 6538, Staphylococcus epidermidis ATCC 12228, Escherichia coli ATCC 25922, Klebsiella pneumoniae ATCC 4352, Pseudomonas aeruginosa ATCC 27853, Proteus mirabilis ATCC 14153 and Candida albicans ATCC 10231 were determined by the microbroth dilutions technique using the Clinical and Laboratory Standards Institute (CLSI) recommendations ${ }^{20,21}$. Mueller-Hinton broth for bacteria, RPMI-1640 medium for yeast strain were used as the test medium. Serial two-fold dilutions ranging from $5000 \mu \mathrm{g} / \mathrm{ml}$ to $4.9 \mu \mathrm{g} / \mathrm{mL}$ were prepared in medium. The inoculum were prepared using a 4-6 h broth culture of each bacteria and $24 \mathrm{~h}$ culture of yeast strains adjusted to a turbidity equivalent to a $0.5 \mathrm{Mc}$ Farland standard, diluted in broth media to give a final concentration of $5 \times 105 \mathrm{cfu} / \mathrm{mL}$ for bacteria and $0.5 \times 103$ to $2.5 \times 103$ $\mathrm{cfu} / \mathrm{mL}$ for yeast in the test tray. The trays were covered and placed in plastic bags to prevent evaporation. The trays containing Mueller-Hinton broth were incubated at $35{ }^{\circ} \mathrm{C}$ for $18-20 \mathrm{~h}$ and the trays containing RPMI-1640 medium were incubated at $35^{\circ} \mathrm{C}$ for $46-50 \mathrm{~h}$. The MIC was defined as the lowest concentration of compound giving complete inhibition of visible growth.

\section{Results and Discussion}

4-Amino-5-(1-phenylethyl)-2,4-dihydro-3H-1,2,4-triazole-3-thione (3) was synthesized by reacting 2-phenylpropanoic acide (1) and thiocarbohydrazide (2) in an oil-bath at $130{ }^{\circ} \mathrm{C}$ for $2 \mathrm{~h}$. The Schiff bases (4a-j) were prepared the synthetic pathway shown in Scheme 1, by reacting equimolar amounts of properly 4-aminotriazole and substituted aldehydes in ethanol over $45 \mathrm{~min}$.<smiles>CC(C(=O)O)c1ccccc1</smiles>

1<smiles>CC(c1ccccc1)c1n[nH]c(=S)n1N</smiles>

3<smiles>NNC(=S)NN</smiles>

2<smiles>CC(c1ccccc1)c1n[nH]c(=S)n1/N=C/[Ga]</smiles>

4a-j

Scheme 1 
The compounds were isolated in satisfactory yields (50-82\%) and purified by recrystallisation, using ethanol. The purities of the synthesized compounds were checked using HPLC. The structures of the synthesized compounds (4a-j) were supported by elemental analysis and by the spectral data achieved from UV, IR, ${ }^{1} \mathrm{H}-\mathrm{NMR}$ and mass spectroscopy which were in agreement with the proposed structures. The characterization data of all the new compounds are summarized in Table 1.

Table 1. Physical and analytical data of compounds (3, 4a-j)

\begin{tabular}{cccccccc}
\hline \multirow{2}{*}{ Comp. } & Molecular & \multicolumn{2}{c}{$\begin{array}{c}\text { M.P. Yield, } \\
\text { formula }\end{array}$} & ${ }^{\circ} \mathrm{C}$ & $\%$ & \multicolumn{4}{c}{ Elemental analysis calculated (found) } \\
\hline $\mathbf{3}$ & $\mathrm{C}_{10} \mathrm{H}_{12} \mathrm{~N}_{4} \mathrm{~S}$ & 174 & 80 & $54.52(54.41)$ & $5.49(5.30)$ & $25.43(24.88)$ & $14.56(14.20)$ \\
$\mathbf{4 a}$ & $\mathrm{C}_{19} \mathrm{H}_{21} \mathrm{~N}_{5} \mathrm{~S}$ & 196 & 50 & $64.93(63.98)$ & $6.02(5.98)$ & $19.93(19.90)$ & $9.12(9.10)$ \\
$\mathbf{4 b}$ & $\mathrm{C}_{19} \mathrm{H}_{20} \mathrm{~N}_{4} \mathrm{O}_{2} \mathrm{~S}$ & 165 & 80 & $61.94(61.90)$ & $5.47(5.32)$ & $15.21(15.10)$ & $8.70(8.35)$ \\
$\mathbf{4 c}$ & $\mathrm{C}_{16} \mathrm{H}_{15} \mathrm{~N}_{5} \mathrm{~S}$ & 182 & 75 & $62.11(62.00)$ & $4.89(4.10)$ & $22.64(22.60)$ & $10.36(10.09)$ \\
$\mathbf{4 d}$ & $\mathrm{C}_{17} \mathrm{H}_{16} \mathrm{~N}_{4} \mathrm{OS}$ & 215 & 80 & $62.94(62.00)$ & $4.97(4.40)$ & $17.27(17.60)$ & $9.88(10.09)$ \\
$\mathbf{4 e}$ & $\mathrm{C}_{17} \mathrm{H}_{15} \mathrm{~N}_{5} \mathrm{O}_{2} \mathrm{~S}$ & 184 & 50 & $57.78(58.01)$ & $4.28(4.25)$ & $19.82(19.80)$ & $9.07(8.90)$ \\
$\mathbf{4 f}$ & $\mathrm{C}_{17} \mathrm{H}_{15} \mathrm{ClN}_{4} \mathrm{~S}$ & 190 & 75 & $59.56(59.41)$ & $4.41(4.30)$ & $16.34(16.88)$ & $9.35(9.20)$ \\
$\mathbf{4 g}$ & $\mathrm{C}_{19} \mathrm{H}_{20} \mathrm{~N}_{4} \mathrm{O}_{2} \mathrm{~S}$ & 190 & 82 & $61.94(61.05)$ & $5.47(5.10)$ & $15.21(15.20)$ & $8.70(8.45)$ \\
$\mathbf{4 h}$ & $\mathrm{C}_{17} \mathrm{H}_{16} \mathrm{~N}_{4} \mathrm{OS}$ & 260 & 65 & $62.94(62.05)$ & $4.97(4.40)$ & $17.27(17.64)$ & $9.88(10.08)$ \\
$\mathbf{4 i}$ & $\mathrm{C}_{17} \mathrm{H}_{15} \mathrm{BrN} \mathrm{N}_{4} \mathrm{~S}$ & 218 & 75 & $52.72(53.00)$ & $3.90(3.98)$ & $14.47(14.90)$ & $8.28(8.10)$ \\
$\mathbf{4 j}$ & $\mathrm{C}_{24} \mathrm{H}_{22} \mathrm{~N}_{4} \mathrm{OS}$ & 230 & 50 & $69.54(68.25)$ & $5.35(4.98)$ & $13.52(13.20)$ & $7.74(7.10)$ \\
\hline
\end{tabular}

In the ${ }^{1} \mathrm{H}$ NMR spectrum, the azomethine protons of compounds $(\mathbf{4 a - j})$ resonated at 9.22-10.27 ppm as expected. Chemical shifts of phenolic protons of compounds $\mathbf{4 d}, \mathbf{4 g}$ and 4h were detected at $9.43,8.55$ and 9.99 ppm respectively. The triazole $\mathrm{NH}$ signal of all synthesized compounds appeared as a singlet at 13.70-14.04 ppm. The aromatic protons were also observed at the expected regions.

Where, Ar: 4-Dimethylaminophenyl (a); 3,4-Dimetoxyphenyl (b); Pyridyl (c); 4Hydroxyphenyl (d); 4-Nitrophenyl (e); 4-Chlorophenyl (f); 3- Ethoxy-4-hydroxyphenyl (g), 2-Hydroxyphenyl (h); 4-Bromophenyl (i); 4-Benzyloxyphenyl (j).

The mass spectroscopic fragmentation of compound $\mathbf{4 b}$ and $\mathbf{4 e}$ were studied under atmospheric pressure electrospray ionization (API-ES). The API-ES spectra of these compounds displayed molecular ions which were confirmed their molecular weights. In the mass spectrum of these compounds, the cleavage of $\mathrm{N}-\mathrm{N}$ bound was observed and substituted triazole fragment was occurred at $m / z 240$.

\section{Antibacterial and antifungal activity}

Antimicrobial activity of novel compounds against Staphylococcus aureus ATCC 6538, Staphylococcus epidermidis ATCC 12228, Escherichia coli ATCC 25922, Klebsiella pneumoniae ATCC 4352, Pseudomonas aeruginosa ATCC 27853, Proteus mirabilis ATCC 14153 and Candida albicans ATCC 10231 were determined by the microbroth dilutions technique using the Clinical and Laboratory Standards Institute (CLSI) recommendations ${ }^{20,21}$.

The minimum inhibitory concentration (MIC) of the synthesized compounds was determined against the bacteria and the yeast $C$. albicans using a standard broth dilution technique. All the MIC results are presented in Table 2. 
Table 2. Antimicrobial activity data of synthesized compounds ${ }^{\mathrm{a}}$

\begin{tabular}{ccccccccccccc}
\hline $\begin{array}{c}\text { Micro- } \\
\text { organism. }\end{array}$ & $\mathbf{3}$ & $\mathbf{4 a}$ & $\mathbf{4 b}$ & $\mathbf{4 c}$ & $\mathbf{4 d}$ & $\mathbf{4 e}$ & $\mathbf{4 f}$ & $\mathbf{4 g}$ & $\mathbf{4 h}$ & $\mathbf{4 i}$ & $\mathbf{4 j}$ & $\begin{array}{c}\text { References, } \\
\text { MIC, } \boldsymbol{\mu g} / \mathrm{mL}\end{array}$ \\
\hline $\mathbf{A}$ & 156 & 312 & 312 & - & 39 & 78 & - & 312 & - & - & 312 & Cef-Na (1.2) \\
$\mathbf{B}$ & 156 & 625 & 625 & - & 39 & 9 & 156 & 625 & 312 & 156 & 625 & Cef-Na (9.8) \\
$\mathbf{C}$ & - & 312 & 9 & 312 & 312 & 625 & 625 & 625 & 312 & 312 & 625 & Cef-Na (4.9) \\
$\mathbf{D}$ & 625 & - & - & - & - & - & - & - & - & - & - & Cef-Na (4.9) \\
$\mathbf{E}$ & - & - & - & - & - & - & - & - & - & 625 & - & Cef-Na (2.4) \\
$\mathbf{F}$ & 78 & 312 & 312 & 312 & 9 & 9 & 156 & 39 & 39 & 39 & 39 & $\mathrm{Ctm}(4.9)$ \\
\hline
\end{tabular}

A: S.aureu, B.: S. Epidermidis, C: E. Coli, D: Klebsiella pneumoniae, E: P. mirabilis, F: C. albicans ${ }^{a}$ The values indicate Minimum Inhibitory Concentrations (MIC) in $\mu \mathrm{g} / \mathrm{ml}$ Cef-Na: Cefuroxime-Na, Cefta: ceftazidime, Ctm: Clotrimazole

The obtained data reported that compounds were able to inhibit the growth of the selected microorganisms in vitro showing MIC values between 9 and $625 \mu \mathrm{g} / \mathrm{mL}$. None of them showed any inhibition against Pseudomonas aeruginosa ATCC 27853 and their results were not shown in Table 2. In general Schiff bases and presence of pharmacophores like $-\mathrm{NO}_{2},-\mathrm{Br}$, phenolic $-\mathrm{OH}$ and $-\mathrm{Cl}$ are reported to posses antimicrobial activities ${ }^{22}$. Among the tested compounds $\mathbf{4 d}$ and $\mathbf{4 e}$ containing 4-OH and 4- $\mathrm{NO}_{2}$ substituted phenyl ring, were found to be the most active derivatives at an MIC value of $9 \mu \mathrm{g} / \mathrm{mL}$ against $C$. albicans. The compound $4 \mathrm{e}$ showed same MIC value $(9 \mu \mathrm{g} / \mathrm{mL})$ as reference compound cefuroxime against $S$. Epidermidis.

\section{Conclusion}

A series of novel Schiff bases have been synthesized and screened for their antimicrobial activities. The antimicrobial screening indicated that among the tested compounds Schiff bases with 4-OH and 4- $\mathrm{NO}_{2}$ substituent exhibited remarkable antimicrobial activity against all the tested microorganism. Thus, the compound $\mathbf{4 d}$ and $\mathbf{4 e}$ having $\mathrm{OH}$ and $\mathrm{NO}_{2}$ substituent could be lead compounds for further molecular modifications in the search for novel antibacterial agents.

\section{References}

1. Patole J, Shingnapurkar D, Padhye S and Ratledge C, Bioorg Med Chem Lett., 2006, 16, 1514.

2. Hearn M J and Cynamon M H, J Antimicrob Chemother., 2004, 53, 185-191.

3. Ren S, Wang R, Komatsu K, Bonaz-Krause P, Zyrianov Y, McKenna C E, Csipke C, Tokes ZA and Lien E J, J Med Chem., 2002, 45, 410-419.

4. Kuz'min V E, Artemenko A G, Lozytska R N, Fedtchouk A S, Lozitsky V P, Muratov E N and Mescheriakov A K, SAR QSAR Environ Res., 2005, 16(3), 219.

5. Ronad P, Dharbamalla S, Hunshal R and Maddi V, Arch Pharm (Weinheim,), 2008, 341(11), 696-700.

6. Bhandari S V, Bothara K G, Raut M K, Patil A A, Sarkate A P and Mokale V J, Bioorg Med Chem., 2008, 16, 1822-1831.

7. Verma M, Pandeya S N, Singh K N and Stables J P, Acta Pharm., 2004, 54(1), 49-56.

8. Sridhar S K, Pandeya S N, Stables J P and Ramesh A, Eur J Pharm Sci., 2002, 16(3), 129-132.

9. Kaplan J P, Raizon B M, Desarmenien M, Feltz P, Headley P M, Worms P, Lloyd K G and Bartholini G, J Med Chem., 1980, 23, 702-704. 
10. Jarrahpour A, Khalili D, De Clercq E, Salmi C and Brunel J M, Molecules, 2007, 12(8), 1720-1730.

11. Shi L, Ge H M, Tan S H, Li H Q, Song Y C, Zhu H L and Tan R X, Eur J Med Chem., 2007, 42(4), 558-564.

12. Holla B S, Veerenda B, Shivananda K and Boja P, Eur J Med Chem., 2003, 38, 759-767.

13. Rollas S, Kalyoncuoğlu N, Sur-Altıner D and Yeğenoğlu Y, Pharmazie, 1993, 48(4), 308-309.

14. Abdel-Megeed A M, Abdel-Rahman H M, Alkaramany G E and El-Gendy M A, Eur J Med Chem., 2009; 44(1), 117-123.

15 Kumar H, Javed S A, Khan S A and Amir M, Eur J Med Chem., 2008, 43(12), 2688-2698.

16. Kaplancikli Z A, Turan-Zitouni G, Ozdemir A and Revial G, Eur J Med Chem., 2008, 43(1), 155-159.

17. Küçükgüzel I, Güniz Küçükgüzel S, Rollas S, Otük-Saniş G, Ozdemir O, Bayrak I, Altuğ T and Stables J P, ll Farmaco, 2004, 59(11), 893-901.

18. Küçükgüzel I, Tatar E, Küçükgüzel S G, Rollas S and De Clercq E, Eur J Med Chem., 2008, 43(2), 381-392.

19. Ashok M, Holla B S and Boja P, Eur J Med Chem., 2007, 42(8), 1095-1101.

20. Clinical and Laboratory Standards Institute (CLSI) Methods for dilution antimicrobial susceptibility tests for bacteria that grow aerobically: Approved Standard 2006; M7A5. Wayne, PA: CLSI.

21. Clinical and Laboratory Standards Institute Reference Method for Broth Dilution Antifungal Susceptibility Testing of Yeasts; Third Informational Supplement 2008; M27-S3. Clinical and Laboratory Standards Institute, Wayne, PA: CLSI.

22. Panneerselvam P, Rather B A, Ravi Sankar Reddy D and Ramesh Kumar N, Eur J Med Chem., 2009, 44(5), 2328-2333. 


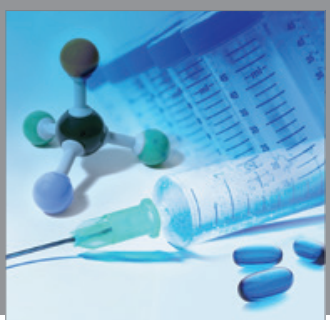

International Journal of

Medicinal Chemistry

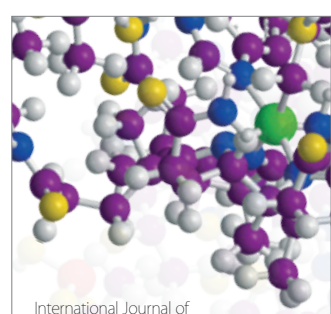

Carbohydrate Chemistry

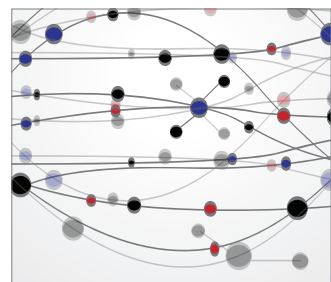

The Scientific World Journal
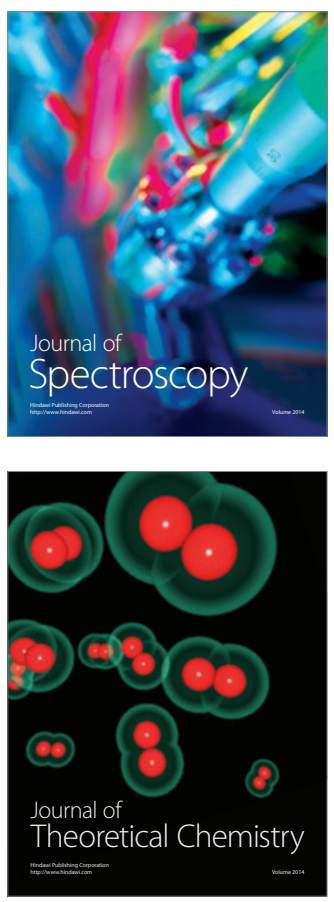
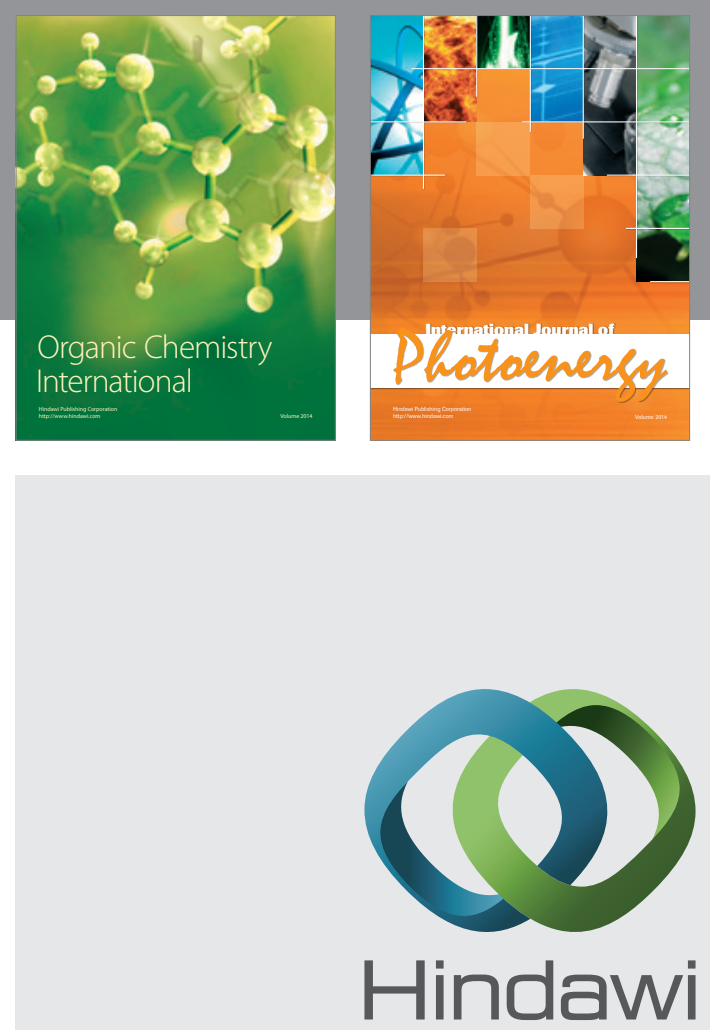

Submit your manuscripts at

http://www.hindawi.com
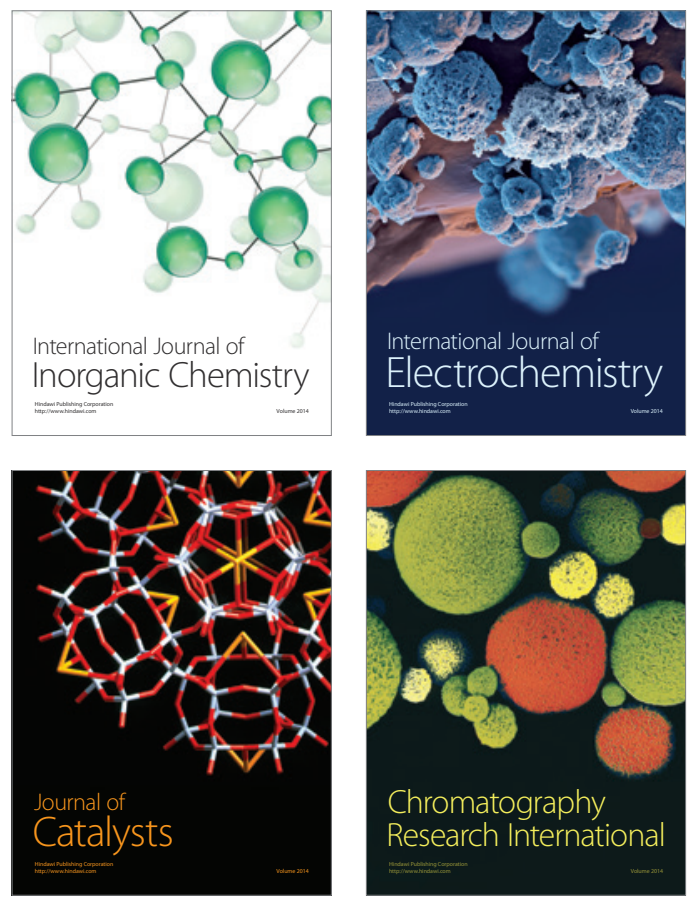
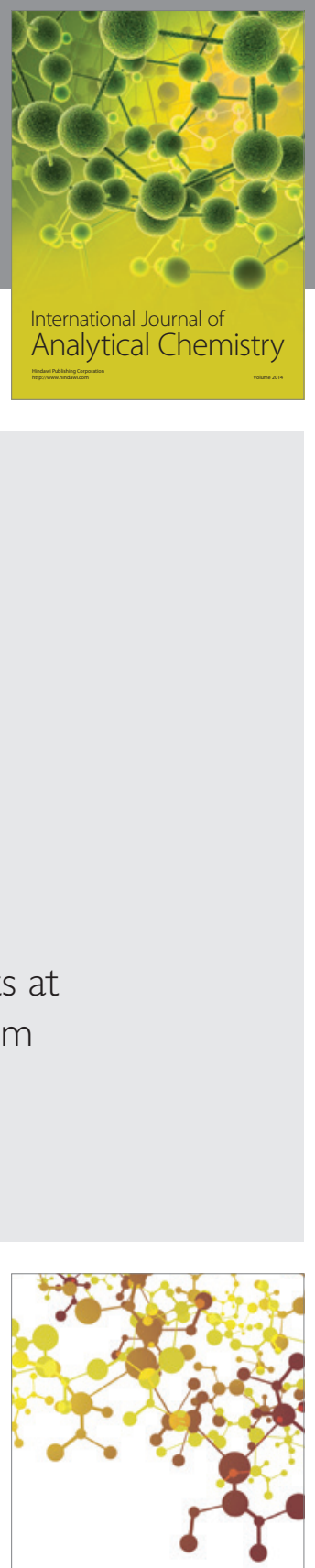

Journal of

Applied Chemistry
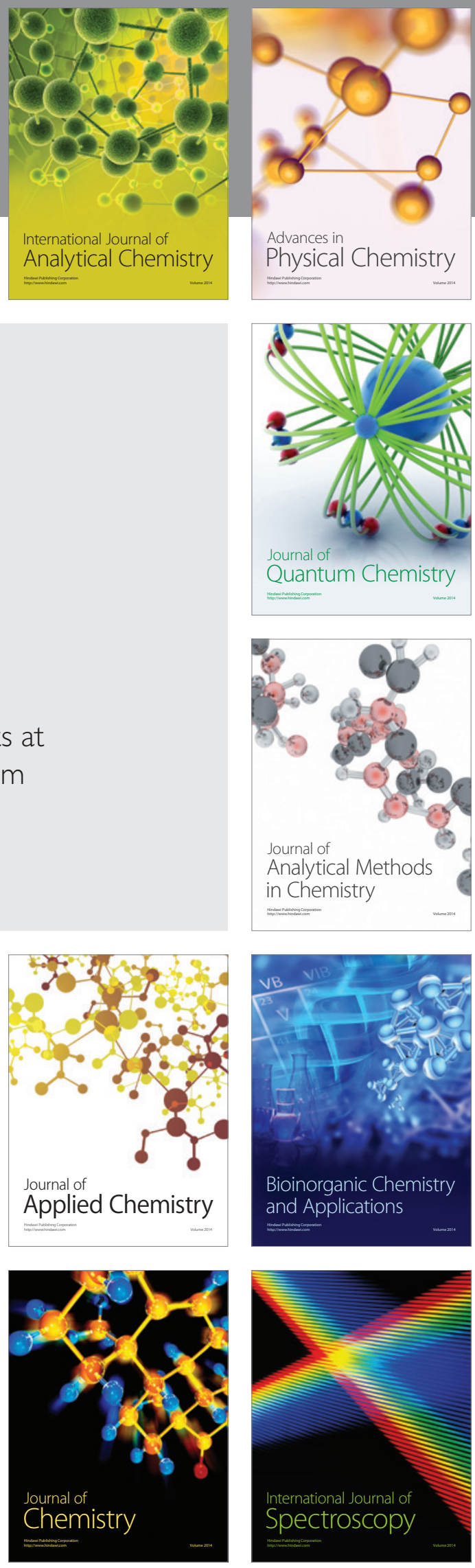